\title{
A fusion protein of hepatocyte growth factor enhances reconstruction of myocardium in a cardiac patch derived from porcine urinary bladder matrix
}

Takeyoshi Ota, MD, PhD, ${ }^{\text {a }}$ Thomas W. Gilbert, PhD, ${ }^{b}$ David Schwartzman, MD, ${ }^{c}$ Charles F. McTiernan, PhD, ${ }^{c}$ Takashi Kitajima, PhD, ${ }^{\mathrm{d}}$ Yoshihiro Ito, PhD, ${ }^{\mathrm{d}}$ Yoshiki Sawa, MD, PhD, ${ }^{\mathrm{e}}$ Stephen F. Badylak, DVM, MD, PhD, ${ }^{\mathrm{b}}$ and Marco A. Zenati, MD ${ }^{\mathrm{a}}$

Objective: We sought to promote myocardial repair using urinary bladder matrix incorporated with a fusion protein that combined hepatocyte growth factor and fibronectin collagen-binding domain in a porcine model. Collagen-binding domain acted as an intermediary to promote hepatocyte growth factor binding and enhance hepatocyte growth factor stability within urinary bladder matrix.

\begin{abstract}
Methods: Urinary bladder matrix incorporated with collagen-binding domain and hepatocyte growth factor was implanted into the porcine right ventricular wall (F group) to repair a surgically created defect. Untreated urinary bladder matrix patches (U group) and Dacron patches (D group) served as controls ( $\mathrm{N}=5 /$ group). Electromechanical mapping was performed 60 days after surgery. Linear local shortening was used to assess regional contractility, and electrical activity was recorded.

Results: Linear local shortening was significantly improved in the F group compared with controls (F: $0.51 \% \pm$ $1.57 \%[P<.05], \mathrm{U}:-1.06 \% \pm 1.84 \%, \mathrm{D}:-2.72 \% \pm 2.59 \%)$, whereas it was inferior to the normal myocardium $(13.7 \% \pm 4.3 \% ; P<.05)$. Mean electrical activity was $1.49 \pm 0.82 \mathrm{mV}$ in the $\mathrm{F}$ group, which was statistically greater than in the control groups $(\mathrm{U}: 0.93 \pm 0.71 \mathrm{mV} ; \mathrm{D}: 0.30 \pm 0.22 \mathrm{mV} ; P<.05)$ and less than the normal myocardium $(8.24 \pm 2.49 \mathrm{mV} ; P<.05)$. Histologic examination showed predominant $\alpha$-smooth muscle actin positive cells with the $\mathrm{F}$ group showing the thickest layer and the $\mathrm{D}$ group showing the thinnest layer, with an endocardial endothelial monolayer. Scattered isolated islands of $\alpha$-actinin positive cells were observed only in the F group, but not in the controls, suggesting the presence of cardiomyocytes.

Conclusion: The collagen-binding domain/hepatocyte growth factor/urinary bladder matrix patch demonstrated increased contractility and electrical activity compared with urinary bladder matrix alone or Dacron and facilitated a homogeneous repopulation of host cells. Urinary bladder matrix incorporated with collagen-binding domain and hepatocyte growth factor may contribute to constructive myocardial remodeling.
\end{abstract}

Biologic scaffold materials derived from naturally occurring extracellular matrix (ECM) have shown the ability to promote site-specific, constructive remodeling in a variety of body systems in both preclinical studies and clinical practice. ${ }^{1,2}$ Recently, an ECM scaffold derived from the basement membrane and lamina propria layers of the porcine urinary bladder, referred to as urinary bladder matrix (UBM), was evaluated for repair of myocardial tissue. UBM showed some promise for myocardial repair, with

\footnotetext{
From the Division of Cardiac Surgery, ${ }^{\mathrm{a}}$ McGowan Institute for Regenerative Medicine, ${ }^{b}$ and Cardiovascular Institute, ${ }^{\mathrm{c}}$ University of Pittsburgh, Pittsburgh, Pa; Nano Medical Engineering Laboratory, RIKEN, The Institute of Physical and Chemical Research, ${ }^{\mathrm{d}}$ Wako, Saitama, Japan; and Department of Cardiovascular Surgery, Osaka University Graduate School of Medicine, ${ }^{\mathrm{e}}$ Suita, Osaka, Japan.

The project described was supported in part by NIHAR053603.

Received for publication March 21, 2008; revisions received May 30, 2008; accepted for publication July 4, 2008.

Address for reprints: Marco A. Zenati, MD, Professor of Surgery and Biomedical Engineering, Division of Cardiac Surgery, University of Pittsburgh, 200 Lothrop St, PUH C-700, Pittsburgh, PA 15213 (E-mail: zenatim@upmc.edu).

J Thorac Cardiovasc Surg 2008;136:1309-17

$0022-5223 / \$ 34.00$

Copyright (c) 2008 by The American Association for Thoracic Surgery

doi:10.1016/j.jtcvs.2008.07.008
}

early regeneration of cardiomyocytes and evidence of electrical activity and mechanical function, but was inferior to normal myocardial tissue 60 days after repair. ${ }^{3-5}$ The purpose of the present study was to determine whether the addition of a biologically active factor, specifically, hepatocyte growth factor (HGF), may accelerate the remodeling response of injured myocardial tissue.

HGF was chosen for investigation in this model because it possesses a potent mitogenic effect on endothelial cells and stimulates angiogenesis. ${ }^{6,7}$ Urbanek and colleagues ${ }^{8}$ reported that the intramyocardial injection of HGF facilitated the migration and proliferation of c-kit positive cells, which differentiate into small, functional myocytes. HGF also enhances "cell-to-cell", and "cell-to-ECM', adhesion through activation of a matrix degradation pathway, which facilitates repopulation of tissues with cells. ${ }^{9}$ In addition, HGF has been shown to suppress fibrosis that is characteristic of maladaptive remodeling of infarcted hearts. ${ }^{10,11}$

The direct addition of HGF to UBM would be expected to yield only transient effects because HGF would likely rapidly elute from the UBM surface. To prolong the presence of HGF in the UBM, a fusion protein combining fibronectin 


$$
\begin{aligned}
& \text { Abbreviations and Acronyms } \\
& \alpha \text {-SMA }=\alpha \text {-smooth muscle actin } \\
& \text { CBD }=\text { collagen-binding domain } \\
& \text { ECM }=\text { extracellular matrix } \\
& \text { HGF }=\text { hepatocyte growth factor } \\
& \text { LLS }=\text { linear local shortening } \\
& \text { MHC }=\text { beta-myosin heavy chain } \\
& \text { RV }=\text { right ventricular } \\
& \text { UBM }=\text { urinary bladder matrix } \\
& \text { VEGF }=\text { vascular endothelial growth factor }
\end{aligned}
$$

collagen-binding domain (CBD) and HGF was used in this study. ${ }^{12}$ CBD has a high affinity exclusively for collagen and gelatin. In a previous study using CBD-HGF, enhanced early endothelialization and site-specific remodeling were observed in a decellularized porcine aortic valve. ${ }^{13}$ We hypothesize that CBD-HGF will alter the phenotypic profile of cells that populate the remodeled UBM cardiac patch and enhance cardiac function.

\section{MATERIALS AND METHODS \\ Urinary Bladder Matrix Scaffold Preparation}

UBM scaffolds were prepared as described previously. ${ }^{3}$ Briefly, the basement membrane of the tunica epithelialis mucosa and the subjacent tunica propria of the porcine urinary bladder, collectively termed "UBM," was mechanically isolated and then decellularized and disinfected by immersion in $0.1 \%(\mathrm{v} / \mathrm{v})$ peracetic acid, $4 \%(\mathrm{v} / \mathrm{v})$ ethanol, and $96 \%(\mathrm{v} / \mathrm{v})$ deionized water for 2 hours. The UBM material was then washed twice for 15 minutes with phosphate-buffered saline $(\mathrm{pH}=7.4)$ and twice for 15 minutes with deionized water.

Four hydrated sheets of UBM were stacked in such a manner that the basement membrane surface was the outermost surface on the top and bottom of the stack. This orientation of sheets was chosen to promote endothelial cell growth on the luminal aspect of the scaffold regardless of the orientation of the scaffold. ${ }^{14}$ The 4-layer construct was laminated by a vacuum-pressing technique. The construct was placed between 2 perforated stainless steel sheets, and the stainless steel plates were placed between sheets of sterile gauze. The entire construct was then sealed in vacuum bagging and subjected to a vacuum of 710 to $730 \mathrm{~mm} \mathrm{Hg}$ for approximately 8 hours. It was finally sterilized by exposure to ethylene oxide. The thickness of each UBM scaffold was approximately $1 \mathrm{~mm}$ in the dry form and $1.5 \mathrm{~mm}$ in the rehydrated form.

\section{Preparation of Collagen-Binding Domain/ Hepatocyte Growth Factor}

CBD was fused to the amino terminus of mature HGF with an inserted amino acid sequence using the baculovirus technique, and CBD-HGF was purified as previously described. ${ }^{12}$ The amount of CBD-HGF present was measured by enzyme-linked immunosorbent assay and expressed as the amount of recombinant HGF (PeproTech, Rocky Hill, NJ).

\section{Incorporation of Collagen-Binding Domain/ Hepatocyte Growth Factor and Urinary Bladder Matrix}

The incorporation of CBD-HGF to UBM was performed under sterile conditions. CBD-HGF $(50 \mu \mathrm{g})$ was incubated at $37^{\circ} \mathrm{C}$ for 15 minutes after adding fetal bovine serum $(20 \mu \mathrm{L})$, which transformed single chains of the CBD-HGF into heterodimers (active form). The UBM scaffolds $(25 \times 25$ $\mathrm{mm})$ were rehydrated with saline and then soaked in an activated CBDHGF solution $(1 \mu \mathrm{g} / \mathrm{mL} ; 25 \mathrm{~mL} /$ patch $)$ for 24 hours at $4^{\circ} \mathrm{C}$. Finally, the CBD-HGF solution was decanted, and the patches were rinsed with phosphate-buffered saline $(50 \mathrm{~mL} /$ patch $)$ before surgery.

\section{Quantitative Determination of Hepatocyte Growth \\ Factor}

The amount of HGF in preimplant CBD-HGF-UBM patches $(\mathrm{N}=5)$ was measured by enzyme-linked immunosorbent assay (HGF Immunoassay Kit, Biosource, Camarillo, Calif) after protein extraction of homogenized patches using a lysis buffer (Tissue Extraction Reagent I, Biosource). Recombinant (r)HGF-UBM patches $(\mathrm{N}=5)$, which were subjected to the same incorporation process except for the use of rHGF (PeproTech) in place of CBD-HGF, and untreated UBM patches $(\mathrm{N}=5)$ served as controls.

\section{Cell Proliferation Assay}

UBM discs ( $5 \mathrm{~mm}$ in diameter, thickness of $0.3 \mathrm{~mm}$ ) were washed with phosphate-buffered saline and then placed in wells of 96-well plates (Nunclon, Nalge Nunc International, Roskilde, Denmark). The discs were incubated for 2 hours at $37^{\circ} \mathrm{C}$ in solutions of CBD-HGF or $\operatorname{rHGF}(0,80$, or 400 $\mathrm{ng} /$ disc in phosphate-buffered saline). Each scaffold was washed several times. Human umbilical vein endothelial cells (Lonza, Walkersville, Md) were seeded (5000 cells/well) and cultured for 7 days in EBM2 medium (Lonza) supplemented with $0.5 \%$ serum and insulin-like growth factor but without other angiogenic factors (endothelial growth factor, fibroblast growth factor-2, and vascular endothelial growth factor [VEGF]). The cell number was measured using WST-1 reagent (Dojindo, Kumamoto, Japan) as previously described. ${ }^{12}$

\section{Myocardial Repair in a Porcine Model}

Yorkshire swine $(40-50 \mathrm{~kg})$ were anesthetized and placed in a left recumbent position. The pericardiotomy was performed, and accordingly the heart was exposed via a right anterolateral thoracotomy in the fourth intercostal space. After the positioning of a tangential clamp on the right ventricle, a small portion in full thickness of the right ventricular (RV) free wall was substituted with a CBD-HGF-UBM patch $(25 \mathrm{~mm}$ in diameter) with a 5-0 continuous polypropylene suture ( $\mathrm{F}$ group, $\mathrm{N}=5$ ) (Figure $1, A$ ). Routine chest closure was performed. At 60 days after implantation, the animals were subjected to electromechanical mapping and euthanized for further examination. Swine similarly receiving untreated UBM (U group, $N=5$ ) or Dacron (thickness of $0.76 \mathrm{~mm}$ ) (D group, $\mathrm{N}=5$ ) were used as controls (Figure $1, B)$. The 60 -day time point was chosen because naturally occurring ECM was typically degraded by this time. ${ }^{15,16}$

The study protocol was approved by the Institutional Animal Care and Use Committee of the University of Pittsburgh. All animals received humane care in compliance with the Guide for the Care and Use of Laboratory Animals, published by the National Institutes of Health (1996).

\section{Electromechanical Mapping}

Electromechanical mapping was performed with the NOGA mapping system (Biosense Webster Inc, Diamond Bar, Calif). The system uses synchronous extracorporeal magnetic fields projected into the thoracic space to determine the location and orientation of a sensor mounted near the distal electrode of a deflectable bipolar mapping catheter (NOGA-Star, Biosense Webster Inc, Diamond Bar, Calif), which can be used to access the endocardial surface. ${ }^{17}$ An intracardiac echocardiography system (AcuNAV; Acuson Siemens, Mountain View, Calif) was use together with NOGA to ensure proper placement of the NOGA-Star catheter. The AcuNAV system consists of a 10F deflectable catheter incorporating a phased-array transducer with programmable operating frequency. ${ }^{18,19}$ Endocardial mapping of the entire endocardial 
CBD-HGF-UBM patch Day\#0

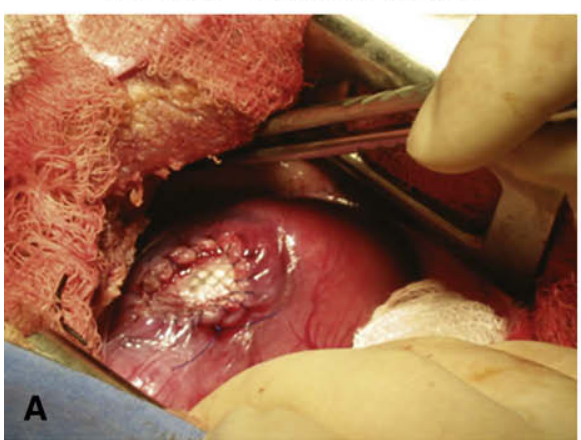

CBD-HGF-UBM patch Day\#60

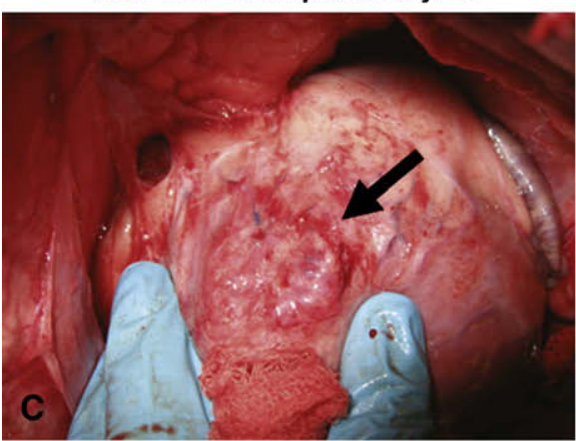

Dacron patch Day\#0

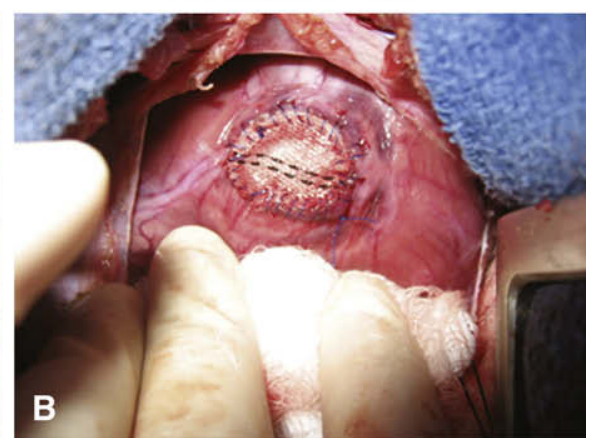

Dacron patch Day\#60

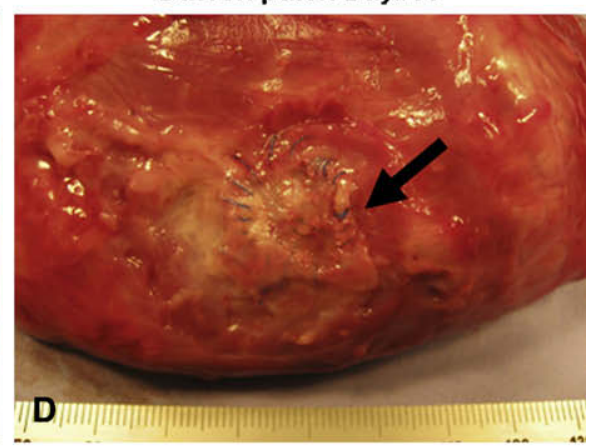

CBD-HGF-UBM patch cross section Day\#60

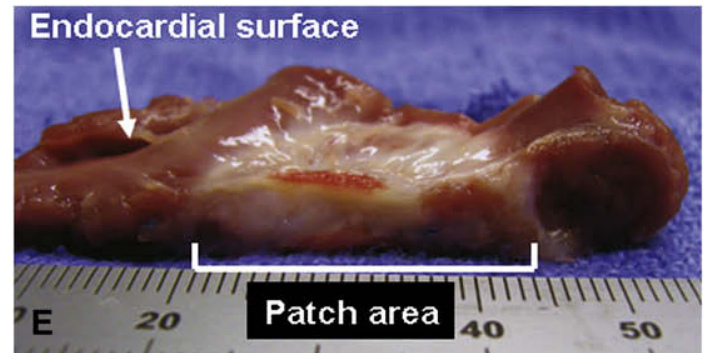

FIGURE 1. Intraoperative view after implantation. A, CBD-HGF-UBM patch. B, Dacron patch. C, CBD-HGF-UBM patch 60 days after implantation. The patch is covered with a thin connective tissue with little adhesion. No aneurysm is seen (arrow). D, Dacron patch 60 days after implantation (excised heart). Severe adhesion (already removed) and deformity of the patch are shown (arrow). E, Cross-section of CBD-HGF-UBM patch. The thickness of the patch area approaches that of the normal myocardium. CBD-HGF-UBM, Collagen-binding domain/hepatocyte growth factor/urinary bladder matrix.

surface was performed during sinus rhythm. The acquired point density for non-free RV walls was $5 / \mathrm{cm}^{2}$; the density for the RV free wall was $10 / \mathrm{cm}^{2}$.

Regional contractility was evaluated by calculating the linear local shortening (LLS) (LLS > 9\%; normal contractility, 9\% > LLS > 0\%; hypokinetic, $0 \%>$ LLS; dyskinetic). The algorithm calculated the fractional shortening of regional endocardial surface at end-systolic phase as previously described. ${ }^{20}$

\section{Quantitative Reverse Transcription-Polymerase Chain Reaction}

Reverse transcription-polymerase chain reaction was performed to quantify the expression of a variety of gene transcripts to have greater insight into the relative presence of various cell types and growth factor expression in the patches at 60 days after implantation ( $\mathrm{N}=5$ for each group), using the TaqMan probe and Applied Biosystems 7700 sequence detector system (Applied Biosystems, Foster City, Calif), as described. ${ }^{21}$ The tissues were taken from the center of the patches and stored in a RNA stabilization reagent (RNAlater, Qiagen, Valencia, Calif). Transcripts that were analyzed included von Willebrand factor, vascular smooth muscle $\alpha$-actin 2, smooth muscle $22 \alpha$, vimentin, beta-myosin heavy chain (MHC), HGF, basic fibroblast growth factor, and VEGF. Beta-MHC was chosen because it was expressed in the early phase of cardiac growth as opposed to alpha-MHC whereas they were both positive in adult hearts. ${ }^{22,23}$ Results were normalized to the level of porcine GAPDH transcripts. Relative expression to GAPDH was quantified with standard curves for both a target gene and GAPDH, ranging from $10^{2}$ to $10^{7}$ copies, which was created for each sample and each assay. The sequences of the specific primers are listed in Table 1.

\section{Histology and Immunohistochemistry}

Excised tissues were fixed in $10 \%$ formalin for paraffin processing or frozen and cut into $5-\mu \mathrm{m}$ sections. The paraffin sections were deparaffinized and stained with hematoxylin-eosin or prepared for immunohistochemical staining. Monoclonal antibodies specific for factor VIII-related antigen (Dako, Carpinteria, Calif) and $\alpha$-smooth muscle actin ( $\alpha$-SMA) (Dako) 
TABLE 1. Primers and probes for quantitative reverse transcriptionpolymerase chain reaction

\begin{tabular}{lll}
\hline GAPDH & Forward & 5'-CTGCACCACCAACTGCTTAGC-3' \\
& Reverse & 5'-GCCATGCCAGTGAGCTTCC-3 \\
& Probe & 5'-CCTGGCCAAGGTCATCCATGACCACTTC-3' \\
vWF & Forward & 5'-ATGGAGTACACGGCTTTGCTG-3' \\
& Reverse & 5'-CAGGCACATGCTGTGACACAT-3' \\
& Probe & 5'-ATGAATGTCCACCTCCTCTTCAGACCGG-3' \\
SM22 $\alpha$ & Forward & 5'-GCTCCATTTGCTTGAAGACCAT-3' \\
& Reverse & 5'-GTAATGCAGTGTGGCCCTGA-3' \\
& Probe & 5'-CTCAAAATCACGCCGTTCTTCAGCCA-3' \\
ACTA22 & Forward & 5'-TATGTGGCTATTCAGGCGGTG-3' \\
& Reverse & 5'-AGGATCTTCATGAGGTAGTCGGTG-3' \\
& Probe & 5'-ATGGTGTCACCCACAACGTGCCCATT-3' \\
Vimentin & Forward & 5'-AGGTGGCAATCTCAATGTCGA-3' \\
& Reverse & 5'-AAATGAGTACCGGAGACAGGTGC-3' \\
& Probe & 5'-CTCTTCCATTTCCCGCATCTGGCGTT-3' \\
MHC & Forward & 5'-CTGAAGGACACCCAGATCCA-3' \\
& Reverse & 5'-GTTGATGAGGCTGGTCTGG-3' \\
& Probe & 5'-ACGCGGTCCGTGCCAATGATGACC-3' \\
HGF & Forward & 5'-ATGATGTCCACGGAAGAGGAGA-3' \\
& Reverse & 5'-CACTCGTAATAGGCCATCATAGTTGA-3' \\
& Probe & 5'-TGCAAACAGGTTCTCAATGTTTCCCAGC- \\
& & 3' \\
bFGF & Forward & 5'-TGTGTGCAAACCGTTATCTTGCTA-3' \\
& Reverse & 5'-CAGTGCCACATACCAACTGGAGTA-3' \\
& Probe & 5'-CTACAATACTTACCGGTCGAGG-3' \\
VEGF & Forward & 5'-GACGTCTACCAGCGCAGCTACT-3' \\
& Reverse & 5'-TTTGATCCGCATAATCTGCATG-3' \\
& Probe & 5'-TTCCAGGAGTACCCCGATGAGATCGA-3' \\
\hline
\end{tabular}

ACTA2, Vascular smooth muscle $\alpha$-actin $2 ; b F G F$, basic fibroblast growth factor; $H G F$, hepatocyte growth factor; $M H C$, beta-myosin heavy chain; $S M 22 \alpha$, smooth muscle $22 \alpha ; V E G F$, vascular endothelial growth factor; $v W F$, von Willebrand factor.

were used. The immunoreaction was detected with 3,3-diaminobenzidine. In addition, cryosections of the tissues were interacted with $\alpha$-sarcomeric actinin (Sigma, St Louis, Mo) and visualized with fluorescein isothiocyanate labeled secondary antibody, using a confocal microscope to detect cardiomyocytes.

\section{Capillary Density}

The factor-VIII positive capillaries were counted under $200 \times$ microscopy. Twenty fields were randomly selected in each patch. Capillary density was expressed as the mean number of vessels per square millimeter. ${ }^{24}$

\section{Statistical Analysis}

All values are expressed as the mean \pm standard deviation. The statistical differences in all data were determined by the Mann-Whitney test.

\section{RESULTS}

\section{In Vitro Analyses}

HGF concentration was $571.8 \pm 246.7 \mathrm{ng} / \mathrm{g}$ dry weight in the CBD-HGF-UBM patches and $163.9 \pm 183.3 \mathrm{ng} / \mathrm{g}$ dry weight in the rHGF-UBM patches $(P<.05)$ (Figure $2, A$ ). HGF was not detected in the untreated UBM patches.

CBD-HGF treatment of the UBM significantly increased cell proliferation compared with HGF alone in both $80 \mathrm{ng} /$ disc $\left(\right.$ CBD-HGF $19.3 \pm 4.4\left[\times 10^{3}\right]$ cells vs HGF $7.9 \pm$ $2.4\left[\times 10^{3}\right]$ cells; $\left.P<.05\right)$ and $400 \mathrm{ng} / \mathrm{disc}(\mathrm{CBD}-\mathrm{HGF}$ $29.5 \pm 2.8\left[\times 10^{3}\right]$ cells vs HGF $20.8 \pm 3.2\left[\times 10^{3}\right]$ cells; $P<.05$ ) (Figure $2, B$ ). The cell proliferation for UBM treated with HGF alone at a concentration of $80 \mathrm{ng} / \mathrm{disc}$ was not significantly different than that of the untreated UBM disc (9.1 $\pm 2.3\left[\times 10^{3}\right]$ cells; $P=.58$ ). In contrast, both treatments with CBD-HGF showed statistically increased cell proliferation compared with the untreated UBM.

\section{Clinical Outcome}

All animals $(\mathrm{N}=15)$ survived with no complications until elective euthanasia at 60 days after initial surgery. No aneurysmal change in the implanted patches was observed in any of the groups at necropsy (Figure 1, C). Connective tissues covered the epicardial side of the patch area with a slight adhesion in the $\mathrm{F}$ and $\mathrm{U}$ groups. Severe adhesion was observed between the Dacron patch and pericardium/lung (D group) with deformity of the patch (Figure 1,D). A thin fibrous encapsulation was present on the endocardial side in the D group that was easily separated from the patch. In the $\mathrm{F}$ and $U$ groups, the patches were completely replaced with a white connective tissue with a thickness comparable to that of the adjacent normal myocardial wall (Figure 1,E).

\section{Electromechanical Mapping}

Local unipolar and bipolar voltage electrograms were recorded. More than 200 discrete points were used to map the right ventricle. Mean electrical activity in the patch regions was $1.49 \pm 0.82 \mathrm{mV}$ in the $\mathrm{F}$ group, which was statistically greater than $0.93 \pm 0.71 \mathrm{mV}$ in the $\mathrm{U}$ group and $0.30 \pm 0.22$ $\mathrm{mV}$ in the $\mathrm{D}$ group $(P<.05)$ and less than the normal myocardium $(8.24 \pm 2.49 \mathrm{mV} ; P<.05)$ (Figure $3, A)$. The LLS was significantly improved in the $\mathrm{F}$ group $(0.51 \% \pm 1.57 \%)$ compared with the U group $(-1.06 \% \pm 1.84 \% ; P<.05)$ and the $\mathrm{D}$ group $(-2.72 \% \pm 2.59 \% ; P<.05)$, whereas they were significantly inferior to the normal myocardium $(13.7 \% \pm$ $4.3 \% ; P<.05$ ) (Figure 3, $B$ ).

\section{Histology and Immunohistochemistry}

Histologic examinations of the F group tissue showed numerous cells through the entire patch, with a monolayer of factor VIII positive cells on the endocardial surface of the remodeled patch (endothelial cells) and predominantly $\alpha$ SMA positive cells homogenously distributed through the entire remodeled patch (Figure 4, $A-C$ ). In the U group, the distribution and phenotypic expression of cells in the remodeled patch, as well as the thickness of the remodeled tissue, were similar to those of the F group, with the exception that $\alpha$-SMA positive cells were predominantly localized at the endocardial aspect and edges of the patch area (Figure 4, $D-F)$. In both the $\mathrm{F}$ and $\mathrm{U}$ groups, there was no UBM remnant visible. In the $\mathrm{D}$ group, although $\alpha$-SMA positive cells were found through the entire patch region, the thickness of the tissue was less than that of the $\mathrm{F}$ or $\mathrm{U}$ group (Figure $4, H$ ). 

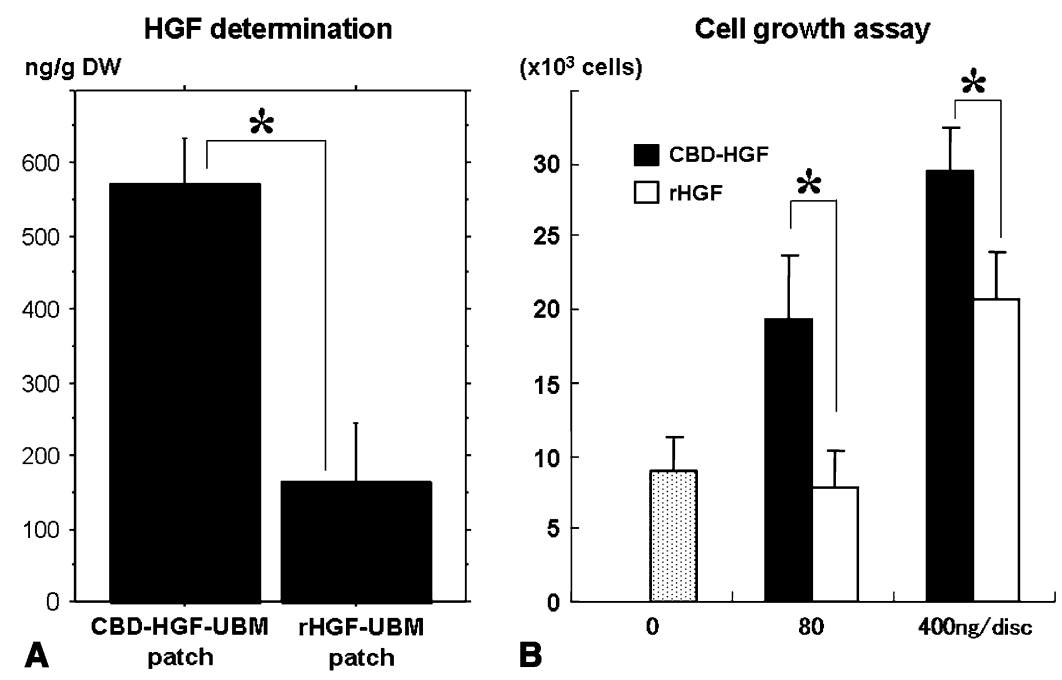

FIGURE 2. In vitro testing. A, HGF determination in preimplant patches. The amount of HGF in the CBD-HGF-UBM patch was significantly higher than that of the rHGF-UBM $(* P<.05)$. B, Growth of human endothelial cells on UBM discs. CBD-HGF shows significantly greater proliferation potential than HGF in each concentration $\left({ }^{*} P<.05\right)$. CBD-HGF-UBM, Collagen-binding domain/hepatocyte growth factor/urinary bladder matrix; $D W$, dry weight; $r H G F$, recombinant hepatocyte growth factor;

The host response to the Dacron patch was consistent with a foreign body response (Figure $4, G$ ). Within the tissue that infiltrated the patch, scattered isolated islands of $\alpha$-sarcomeric actinin positive cells were observed in the $\mathrm{F}$ group ( $\sim 17.1 \%$ of the tissue fields of the entire patch region in the F group showed the presence of $\alpha$-actinin positive cells), but not in the $\mathrm{U}$ and $\mathrm{D}$ groups (Figure 4,I).

\section{Capillary Density}

The capillary density was the highest in the F group ( 31.7 $\pm 20.0 / \mathrm{mm}^{2}$ ) with a statistically significant difference, compared with $14.3 \pm 10.5 / \mathrm{mm}^{2}$ in the U group and $4.6 \pm 4.4$ / $\mathrm{mm}^{2}$ in the D group (Figure 5). A common characteristic in the 3 groups was that the capillary structures were predominantly distributed in epicardial aspect of the patches.

\section{Quantitative Real-Time Reverse Transcription- Polymerase Chain Reaction}

mRNA expression was normalized by the expression of a housekeeping gene (GAPDH). The results are summarized in Table 2 and Figure 6. In the $\mathrm{F}$ and $\mathrm{U}$ groups, the early mesenchymal markers (ie, vascular smooth muscle $\alpha$-actin 2, smooth muscle $22 \alpha$, vimentin) were expressed significantly more than in the normal myocardium. There was also higher expression of mesenchymal markers in the $\mathrm{F}$ group compared with the $\mathrm{U}$ group. Expression of
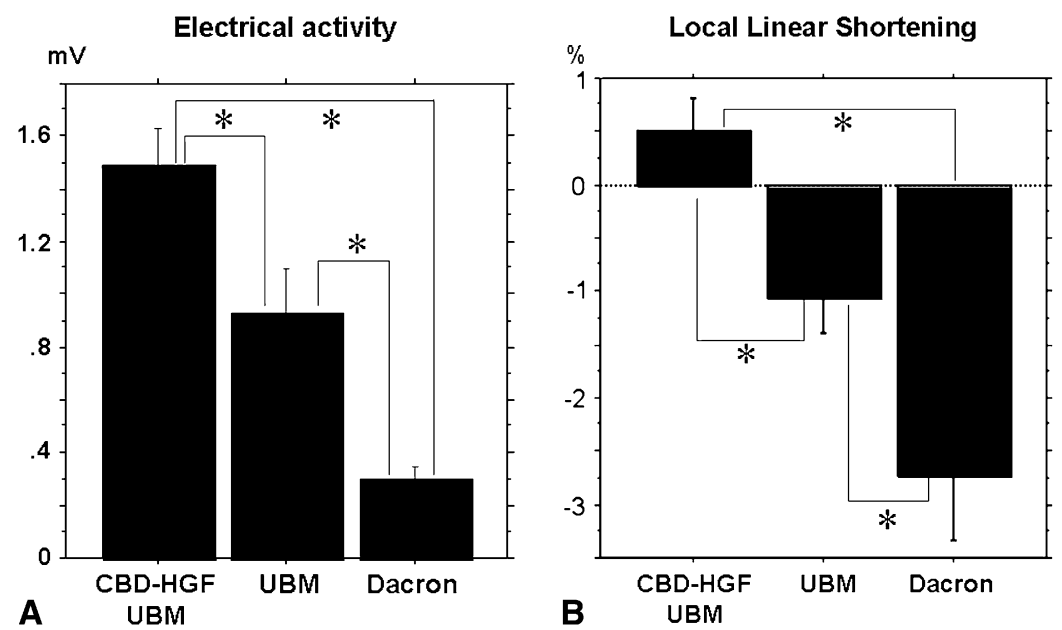

FIGURE 3. A, Electrical activity in the patches. CBD-HGF-UBM patch shows the greatest electrical activity compared with the others $(* P<.05)$. B, LLS of the patch regions. CBD-HGF-UBM patch is positively contractile, whereas the UBM and Dacron patches are dyskinetic $\left({ }^{*} P<.05\right)$. CBD-HGF-UBM, Collagen-binding domain/hepatocyte growth factor/urinary bladder matrix. 


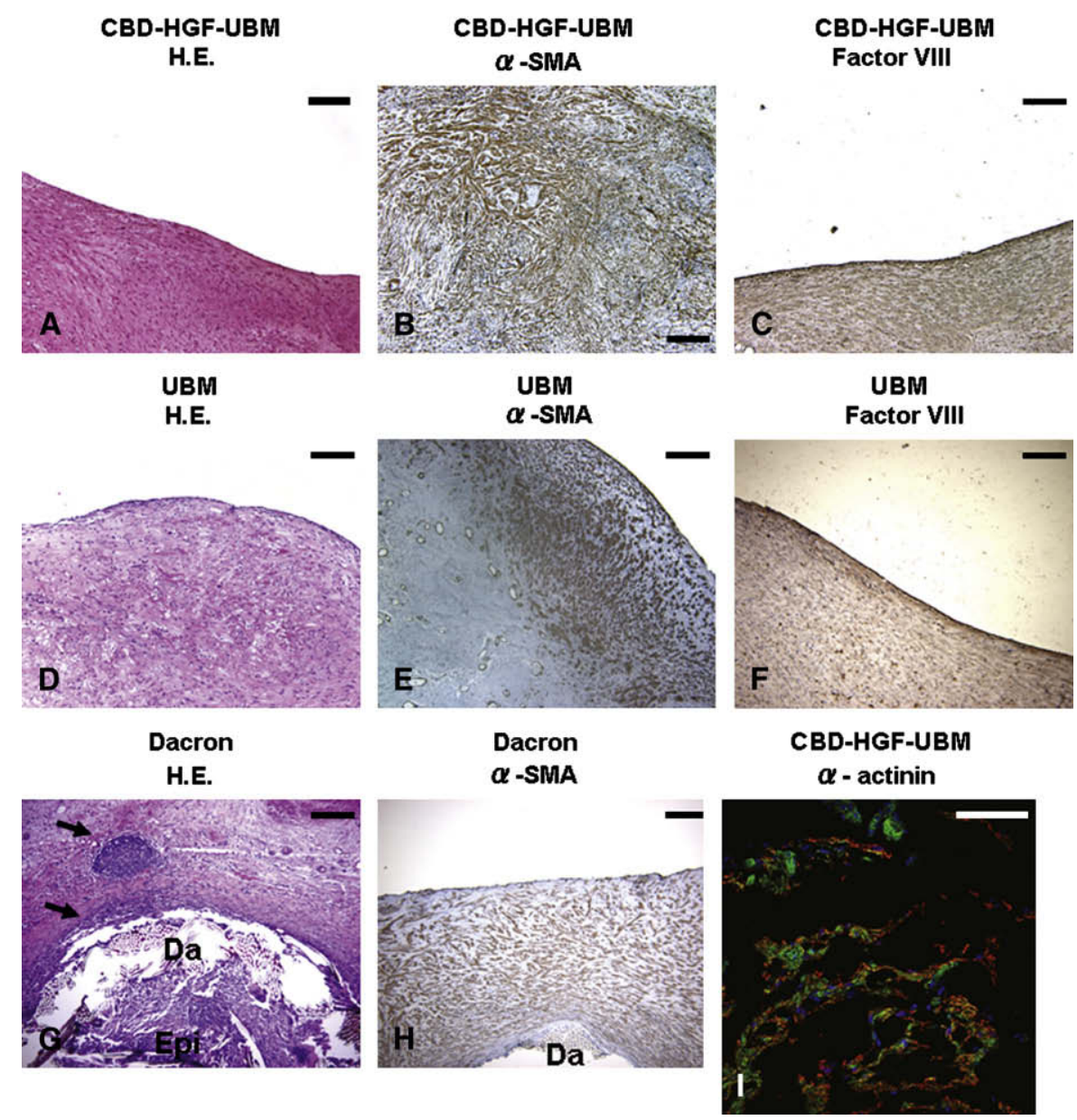

FIGURE 4. Histologic preparation of the patches 60 days after implantation. A, B, C, CBD-HGF-UBM patches show a monolayer of factor VIII positive cells, and predominantly $\alpha$-SMA positive cells homogenously spread to the entire patch area beneath the endothelial monolayer. D, E, F, UBM patches show $\alpha$-SMA positive cells predominantly distributed in the endocardial aspect and the edge of the patch area with an endocardial monolayer of factor VIII-positive cells. G, H, Thickness of $\alpha$-SMA positive cells is less than that of the CBD-HGF-UBM or UBM patches. Inflammatory reaction was seen along the Dacron patch (arrow). I, CBD-HGF-UBM patch stained with $\alpha$-sarcomeric actinin stain. Blue, green, and red indicate nuclei, phalloidin, and $\alpha$-sarcomeric actinin, respectively. A, D, G, Hematoxylin-eosin stain. B, E, H, $\alpha$-SMA. C, F, Factor VIII. I, $\alpha$-sarcomeric actinin. Black bar $=200 \mu \mathrm{m}(\times 100)$. White bar $=100 \mu \mathrm{m}$ (×200). Da, Dacron; Epi, epicardial side; CBD-HGF-UBM, collagen-binding domain/hepatocyte growth factor/urinary bladder matrix; H.E., hematoxylineosin; SMA, smooth muscle actin.

von Willebrand factor was similar in the $\mathrm{F}$ and $\mathrm{U}$ groups and was not statistically different from the normal myocardium. In contrast, the expression levels of von Willebrand factor, vascular smooth muscle $\alpha$-actin 2, smooth muscle $22 \alpha$, and vimentin were significantly higher in the D group than in the other groups, including the normal myocardium, which is consistent with the hyperplasic scar formation observed histologically in the D group. The expression of $\mathrm{MHC}$ in the $\mathrm{F}$ and $\mathrm{U}$ groups was detected with significantly higher level in the F group, whereas it was not detected in the D group. Both VEGF and basic fibroblast growth factor were expressed significantly more in the $\mathrm{F}$ and $\mathrm{U}$ groups than in the D group. The expression of HGF was greater in the $\mathrm{F}$ group than in the $\mathrm{U}$ group $(P<.05)$, whereas there was no HGF detected in the normal myocardium and the D group.

\section{DISCUSSION}

The present study showed that the addition of CBD-HGF to UBM increased proliferation of endothelial cells to a greater extent than the untreated UBM. Histologic analyses showed that the implanted CBD-HGF-UBM patches resulted in constructive remodeling consisting of a monolayer of endothelial cells, well-organized myofibroblasts, and scattered cells that are tentatively identified as cardiomyocytes. One of the most important findings in this study was that the CBD-HGF-UBM patch showed an improved recovery of mechanical and electrophysiologic functions. The CBD-HGF-UBM patches were positively contractile, whereas the untreated UBM and Dacron patches were dyskinetic. Likewise, recovery of the electrical activity in the patches was the greatest in the CBD-HGF-UBM, although it was far less than in the normal myocardium. 


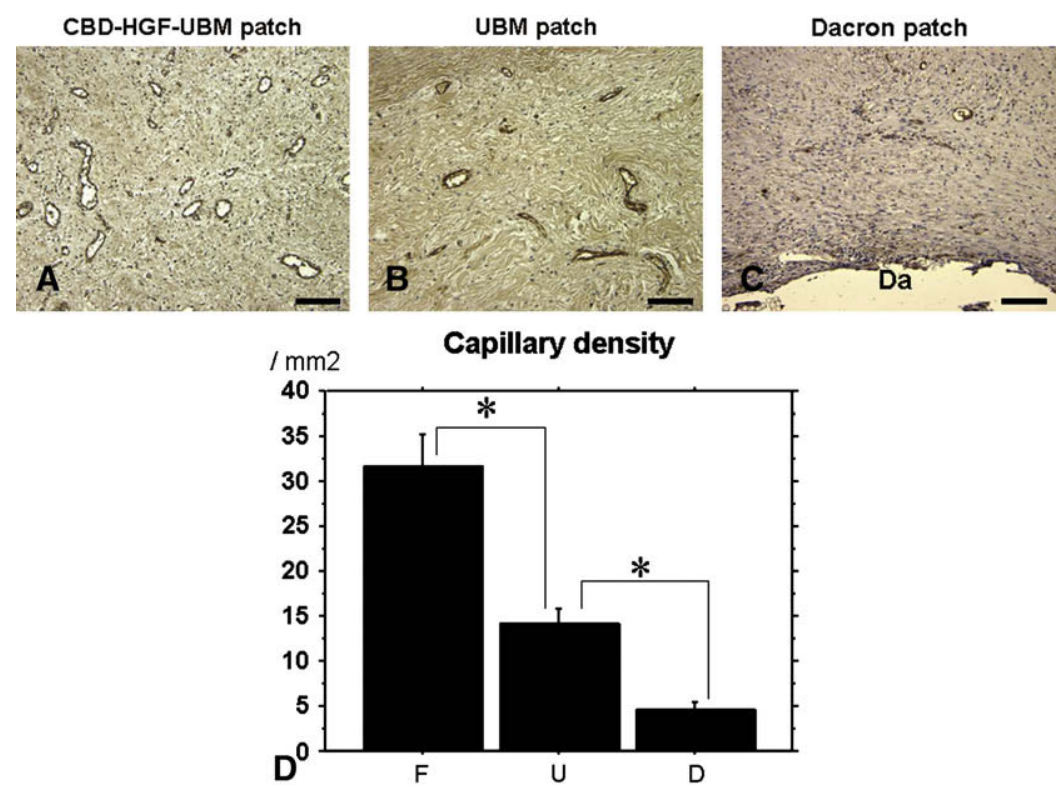

FIGURE 5. Capillary density and representative images. Representative factor VIII staining in each patch for capillary density study: A, CBD-HGF-UBM patch. B, UBM patch. C, Dacron patch. Black bar $=100 \mu \mathrm{m}$. D, Capillary density. CBD-HGF-UBM patch shows highest capillary density of all $(* P<.05)$. F, CBD-HGF-UBM patch group. $U$, UBM patch group, $D$, Dacron patch group. $D a$, Dacron patch; $C B D-H G F-U B M$, collagen-binding domain/hepatocyte growth factor/urinary bladder matrix.

The morphologic observations and gene expression analyses suggest that the reparative pathways for untreated UBM patches and CBD-HGF-UBM patches were the same, but that the rate of repair occurred more slowly for the untreated UBM. In contrast, the host response to the Dacron patch was more consistent with a chronic foreign body hyperplastic response with scar tissue formation and the presence of inflammatory cells. The results of histology and reverse transcription-polymerase chain reaction indicate that the repaired tissues in the untreated UBM and CBD-HGF-UBM patches are different from the typical myocardial scar. For example, healed wound tissue from the Dacron patch-treated hearts had no evidence for MHC gene expression or $\alpha$-actinin positive cells, markers that are commonly used to identify cardiomyocytes. The high expression of growth factors (ie, VEGF, basic fibroblast growth factor, HGF) in the CBD-
HGF-UBM and untreated UBM patches compared with those in normal myocardium and Dacron patches suggests that cells present in the UBM patches are participating in the remodeling response.

The results emphasize the importance of rapid and aggressive angiogenesis in the remodeled scaffold. In the untreated UBM, the repopulated cells are localized on the endocardial aspect and at the edges of the patch where blood supplies exist. The more uniform distribution of cells throughout the remodeled CBD-HGF-UBM was associated with an increased vascularity that implied increased blood supply. The mechanisms by which HGF promotes angiogenesis and the source of the cells that populate the scaffold remain unclear. However, evidence shows a circulating source of progenitor cells, including cells from the bone marrow, that is recruited to the site of ECM remodeling. ${ }^{25-27}$

TABLE 2. Results of quantitative real-time reverse transcription-polymerase chain reaction

\begin{tabular}{lcccc}
\hline & Normal myocardium & CBD-HGF-UBM (F) & UBM (U) & Dacron (D) \\
\hline ACTA2 & $0.13 \pm 0.04$ & $0.58 \pm 0.45^{\mathrm{a}, \mathrm{b}, \mathrm{c}}$ & $0.25 \pm 0.25^{\mathrm{c}}$ & $1.32 \pm 0.98^{\mathrm{a}}$ \\
SM22 $\alpha$ & $0.032 \pm 0.034$ & $0.81 \pm 0.61^{\mathrm{a}, \mathrm{b}, \mathrm{c}}$ & $0.23 \pm 0.22^{\mathrm{a}, \mathrm{c}}$ & $1.09 \pm 0.98^{\mathrm{a}, \mathrm{c}}$ \\
Vimentin & $0.11 \pm 0.03$ & $1.39 \pm 1.03^{\mathrm{a}, \mathrm{c}}$ & $0.27 \pm 0.07^{\mathrm{c}}$ & $3.15 \pm 2.47^{\mathrm{a}}$ \\
VWF & $0.23 \pm 0.11$ & $0.32 \pm 0.19^{\mathrm{c}}$ & $0.28 \pm 0.37^{\mathrm{a}}$ & $1.47 \pm 1.84^{\mathrm{a}}$ \\
MHC & $5.82 \pm 1.24$ & $1.65 \pm 0.67^{\mathrm{a}, \mathrm{b}}$ & $15.46 \pm 8.77^{\mathrm{c}}$ & - \\
VEGF $\left(\times \mathbf{1 0}^{\mathbf{4}}\right)$ & $9.68 \pm 11.27$ & $14.97 \pm 11.4^{\mathrm{c}}$ & $282 \pm 260^{\mathrm{a}, \mathrm{c}}$ & $3.14 \pm 3.36^{\mathrm{a}}$ \\
bFGF $\left(\times \mathbf{1 0}^{-\mathbf{4}}\right)$ & $2.31 \pm 1.82$ & $339 \pm 198^{\mathrm{a}, \mathrm{c}}$ & $0.6 \pm 0.27$ & $-2.4 \pm 24.2^{\mathrm{a}}$ \\
HGF $\left(\times \mathbf{1 0}^{-\mathbf{4}}\right)$ & - & $2.24 \pm 0.82$ & - \\
\hline
\end{tabular}

$\overline{A C T A 2}$, Vascular smooth muscle $\alpha$-actin $2 ; b F G F$, basic fibroblast growth factor; $H G F$, hepatocyte growth factor; $M H C$, beta-myosin heavy chain; $S M 22 \alpha$, smooth muscle $22 \alpha$; $V E G F$, vascular endothelial growth factor; $v W F$, von Willebrand factor. Expressed with the mRNA level/GAPDH. $P<.05 .{ }^{\mathrm{a}}$ Versus normal myocardium. ${ }^{\mathrm{b}} \mathrm{Versus}$ UBM (U). ${ }^{\mathrm{c}} \mathrm{Ver}-$ sus Dacron (D). 


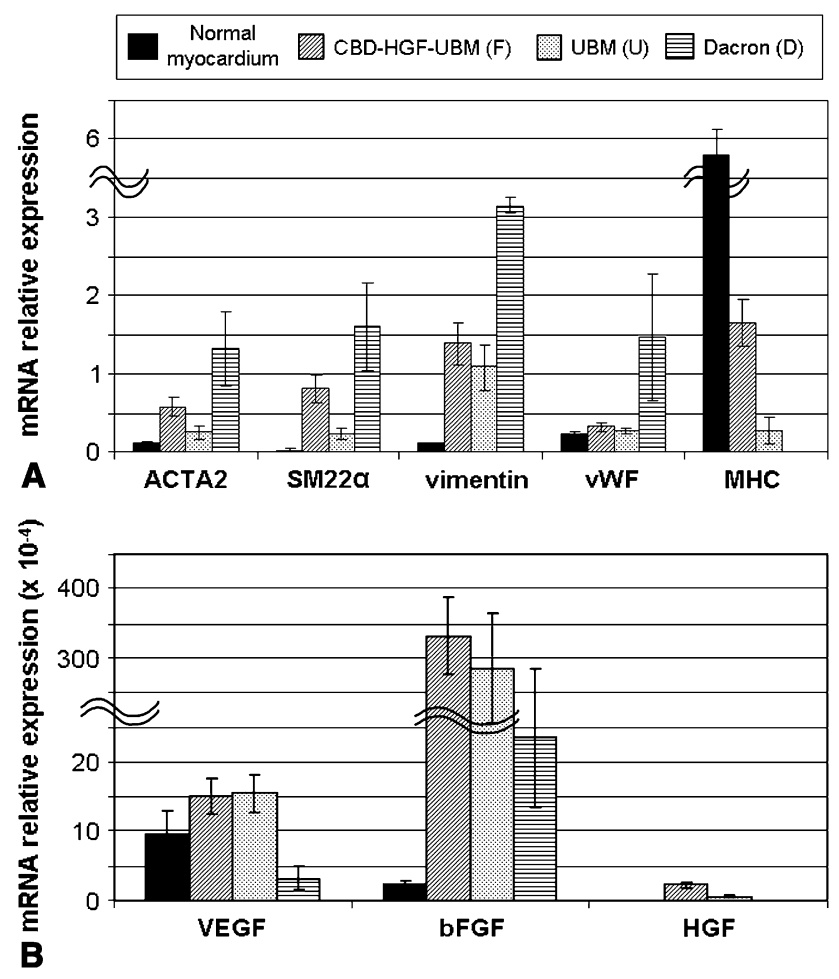

FIGURE 6. Graphs of quantitative real-time reverse transcription-polymerase chain reaction. $C B D-H G F-U B M$, Collagen-binding domain/hepatocyte growth factor/urinary bladder matrix; $A C T A 2$, vascular smooth muscle $\alpha$-actin $2 ; b F G F$, basic fibroblast growth factor; $M H C$, beta-myosin heavy chain; $S M 22 \alpha$, smooth muscle $22 \alpha ; V E G F$, vascular endothelial growth factor; $v W F$, von Willebrand factor.

Recent studies have shown that non-cross-linked ECM scaffolds promote an anti-inflammatory, accommodative immune response, with an accumulation of macrophages expressing the M2 phenotype. $^{28}$ The treatment of ECM scaffolds and tissues with crosslinking agents has been advocated to slow degradation and increase mechanical integrity of the graft. The crosslinking process necessarily eliminates the release of bioactive peptides that are beneficial to the remodeling response (eg, bacteriostasis, chemotaxis, angiogenesis). Furthermore, chemically cross-linked ECM scaffolds induce macrophages to express a proinflammatory phenotype that results in increased fibrous connective tissue formation. ${ }^{28}$

\section{Study Limitations}

There are several limitations to the present study. First, no effort was made to examine electrical couplings and gap junctions to prove regeneration of the conducting system. Given the nascent signs of electrical remodeling (ie, sparse myocytes, significant but slight electrical activity in the patches), however, those electrical signals might be attributed to the aggregation of electrophysiologic activity by immature mesenchymal cells. ${ }^{29,30}$ Second, the study only evaluated a single time point. It is not clear how the remod- eling progresses in the period immediately after implantation or in the long term, which should be investigated to fully characterize the remodeled tissues. However, it is known that naturally occurring ECM scaffolds degrade within 60 to 90 days after implantation, ${ }^{15,16}$ so any subsequent adaptations of the myocardium would likely be a consequence of remodeling in response to local environmental cues. Third, an RV model was used to decrease mortality and morbidity for the animal, and as a comparison with previous results. Although a UBM patch manufactured by the identical method described for the control patch has been shown to be effective and durable for repair of the left ventricle, ${ }^{4}$ further studies including the left ventricular model and a diseased model (ie, infarction) are needed to determine the efficacy of the CBD-HGF treatment to UBM. It is also important to test our patch in an infarcted heart model because blood supplies to the implanted patch from the surrounding tissue would be significantly reduced or completely shut off, which might negatively influence myocardial remodeling in the patch. Fourth, we did not investigate the impact of the remodeled tissues on the global cardiac function. Given the results of electrical activity and regional contractility in this study, the functional contribution of the patch area might be insignificant at 60 days after implantation. To make it clear, we plan to use a catheter-based pressure-volume assessment at sequential time points in the future study. Likewise, it is important to evaluate the synchronization between the remodeled tissue and the surrounding myocardium using such an echocardiography. Finally, the gene expression and immunofluorescent staining are consistent with the appearance of cells with cardiomyocyte characteristics in the cardiac wounds repaired with CBD-HGF-UBM. The current results cannot prove whether the HGF-UBM patch actually stimulated cardiomyocyte regeneration in the cardiac wound. Thus, a more rigorous analysis of additional cardiomyocyte and cell proliferation markers will be necessary to definitively identify the presence of newly regenerated cardiomyocytes in these repaired wounds.

\section{CONCLUSIONS}

The CBD-HGF-UBM patch demonstrated significantly increased contractility and electrical activity compared with UBM alone or Dacron, and facilitated a homogeneous repopulation of host cells that may include cardiomyocytes. UBM incorporated with CBD-HGF may contribute to sitespecific constructive myocardial remodeling as a substitute for synthetic materials that are currently used for applications such as surgical ventricular restoration.

The expert assistance of Kazuro L. Fujimoto, MD, PhD, Kimimasa Tobita, MD, Gregory Gibson, BS, Jennifer Gabany, MSN, CRNP-C, CCRC, Stacy Cashman, LVT, David Fischer, BS, Melanie O'Malley, LVT, and Masako Yokoyama, BS, is gratefully acknowledged. 


\section{References}

1. Badylak SF. Xenogeneic extracellular matrix as a scaffold for tissue reconstruction. Transplant Immunol. 2004;12:367-77.

2. Ota T, Taketani S, Iwai S, Miyagawa S, Furuta M, Hara M, et al. Novel method of decellularization of porcine valves using polyethylene glycol and gamma irradiation. Ann Thorac Surg. 2007;83:1501-7.

3. Kochupura PV, Azeloglu EU, Kelly DJ, Doronin SV, Badylak SF, Krukenkamp IB, et al. Tissue-engineered myocardial patch derived from extracellular matrix provides regional mechanical function. Circulation. 2005; 112(Suppl):I144-9.

4. Robinson KA, Li J, Mathison M, Redkar A, Cui J, Chronos NA, et al. Extracellular matrix scaffold for cardiac repair. Circulation. 2005;112(suppl): I135-43.

5. Ota T, Gilbert TW, Badylak SF, Schwartzman D, Zenati MA. Electromechanical characterization of a tissue-engineered myocardial patch derived from extracellular matrix. J Thorac Cardiovasc Surg. 2007;133:979-85.

6. Bussolino F, Di Renzo MF, Ziche M, Bocchietto E, Olivero M, Naldini L, et al. Hepatocyte growth factor is a potent angiogenic factor which stimulates endothelial cell motility and growth. J Cell Biol. 1992;119:629-41.

7. Morishita R, Aoki M, Yo Y, Ogihara T. Hepatocyte growth factor as cardiovascular hormone: role of HGF in the pathogenesis of cardiovascular disease. Endocr J. 2002;49:273-84.

8. Urbanek K, Rota M, Cascapera S, Bearzi C, Nascimbene A, De Angelis A, et al. Cardiac stem cells possess growth factor-receptor systems that after activation regenerate the infarcted myocardium, improving ventricular function and longterm survival. Circ Res. 2005;97:663-73.

9. Trusolino L, Cavassa S, Angelini P, Andó M, Bertotti A, Comoglio PM, et al. $\mathrm{HGF} / \mathrm{scatter}$ factor selectively promotes cell invasion by increasing integrin avidity. FASEB J. 2000;14:1629-40.

10. Takimoto Y, Teramachi M, Okumura N, Nakamura T, Shimizu Y. Relationship between stenting time and regeneration of neoesophageal submucosal tissue. ASAIO J. 1994;40:M793-7.

11. Miyagawa S, Sawa Y, Taketani S, Kawaguchi N, Nakamura T, Matsuura N, et al. Myocardial regeneration therapy for heart failure: hepatocyte growth factor enhances the effect of cellular cardiomyoplasty. Circulation. 2002;105: 2556-61.

12. Kitajima T, Terai H, Ito Y. A fusion protein of hepatocyte growth factor for immobilization to collagen. Biomaterials. 2007;28:1989-97.

13. Ota T, Sawa Y, Iwai S, Kitajima T, Ueda Y, Coppin C, et al. Fibronectin-hepatocyte growth factor enhances reendothelialization in tissue-engineered heart valve. Ann Thorac Surg. 2005;80:1794-801.

14. Brown B, Lindberg K, Reing J, Stolz DB, Badylak SF. The basement membrane component of biologic scaffolds derived from extracellular matrix. Tissue Eng. 2006;12:519-26.

15. Gilbert TW, Stewart-Akers AM, Simmons-Byrd A, Badylak SF. Degradation and remodeling of small intestinal submucosa in canine Achilles tendon repair. J Bone Joint Surg Am. 2007;89:621-30.
16. Record RD, Hillegonds D, Simmons C, Tullius R, Rickey FA, Elmore D, et al. In vivo degradation of 14C-labeled small intestinal submucosa (SIS) when used for urinary bladder repair. Biomaterials. 2001;22:2653-9.

17. Gepstein L, Hayam G, Shpun S, Ben-Haim SA. Hemodynamic evaluation of the heart with a nonfluoroscopic electromechanical mapping technique. Circulation. 1997;96:3672-80.

18. Packer DL, Stevens CL, Curley MG, Bruce CJ, Miller FA, Khandheria BK, et al. Intracardiac phased-array imaging: methods and initial clinical experience with high resolution, under blood visualization: initial experience with intracardiac phased-array ultrasound. J Am Coll Cardio1. 2002;39:509-16.

19. Schwartzman D, Bonanomi G, Zenati MA. Epicardium-based left atrial ablation: impact on electromechanical properties. J Cardiovasc Electrophysiol. 2003;14: 1087-92.

20. Gepstein L, Goldin A, Lessick J, Hayam G, Shpun S, Schwartz Y, et al. Electromechanical characterization of chronic myocardial infarction in the canine coronary occlusion model. Circulation. 1998;98:2055-64.

21. Iwai S, Sawa Y, Taketani S, Torikai K, Hirakawa K, Matsuda H. Novel tissue-engineered biodegradable material for reconstruction of vascular wall. Ann Thorac Surg. 2005;80:1821-7.

22. Lyons GE, Schiaffino S, Sassoon D, Barton P, Buckingham M. Developmental regulation of myosin gene expression in mouse cardiac muscle. $J$ Cell Biol. 1990;111:2427-36

23. Chang KC, Figueredo VM, Schreur JH, Kariya K, Weiner MW, Simpson PC et al. Thyroid hormone improves function and $\mathrm{Ca} 2+$ handling in pressure overload hypertrophy. Association with increased sarcoplasmic reticulum $\mathrm{Ca} 2+-\mathrm{ATPase}$ and alpha-myosin heavy chain in rat hearts. J Clin Invest. 1997;100:1742-9.

24. Kobayashi T, Hamano K, Li TS, Katoh T, Kobayashi S, Matsuzaki M, et al Enhancement of angiogenesis by the implantation of self bone marrow cells in a rat ischemic heart model. J Surg Res. 2000;89:189-95.

25. Brountzos E, Pavcnik D, Timmermans HA, Corless C, Uchida BT, Nihsen ES et al. Remodeling of suspended small intestinal submucosa venous valve: an experimental study in sheep to assess the host cells' origin. $J$ Vasc Interv Radiol. 2003; 14:349-56.

26. Badylak SF, Park K, Peppas N, McCabe G, Yoder M. Marrow-derived cells populate scaffolds composed of xenogeneic extracellular matrix. Exp Hematol. 2001; 29:1310-8.

27. Zantop T, Gilbert TW, Yoder MC, Badylak SF. Extracellular matrix scaffolds are repopulated by bone marrow-derived cells in a mouse model of Achilles tendon reconstruction. J Orthop Res. 2006;24:1299-309.

28. Badylak SF, Gilbert TW. Immune response to biologic scaffold materials. Semin Immunol. 2008;20:109-16. Epub 2008 Feb 20. Review.

29. Heubach JF, Graf EM, Leutheuser J, Bock M, Balana B, Zahanich I, et al. Electrophysiological properties of human mesenchymal stem cells. J Physiol. 2004; 554:659-72.

30. Halbach M, Pfannkuche K, Pillekamp F, Ziomka A, Hannes T, Reppel M, et al Electrophysiological maturation and integration of murine fetal cardiomyocytes after transplantation. Circ Res. 2007;101:484-92. 